\section{NOUVEAUX PROCÉDÉS \\ de préparation de surface par laser}

Bruno RAMEAU ${ }^{1}$, Sophie PETTIER ${ }^{1}$, Guillaume LAFOY ${ }^{2}$, Charly LOUMENA², Rainer KLING

Airbus Safran Launchers,

rue du Général Niox, BP 20011, 33165 Saint-Médard-en-Jalles Cedex, France

ALPHANOV, Institut d'optique d'Aquitaine,

rue François Mitterrand, 33400 Talence, France

Le laboratoire matériaux et procédés d'Airbus Safran Launchers à Saint-Médard-en-Jalles développe et met au point de nouvelles technologies pour améliorer les procédés de mise en œuvre et de traitement des matériaux. L'objectifest de favoriser l'émergence de technologies à faible impact sur l'environnement et à coûts et délais de mise en œuvre réduits. C'est dans ce cadre que le laboratoire a travaillé en collaboration avec ALPhANOV, le centre technologique du pôle de compétitivité Route des Lasers. Les travaux portent sur l'évaluation et la mise au point de sources laser pour améliorer les propriétés et performances de matériaux et structures mis en œuvre lors d'opérations de préparation de surface avant collage ou avant protection contre la corrosion.

\section{Problématique et solutions}

Le collage et la protection anticorrosion sont des éléments cruciaux dans l'industrie aéronautique afin de garantir la sécurité et la fiabilité dans le temps des pièces utilisées. Les technologies employées actuellement, en bain pour la plupart, donnent des résultats satisfaisants, mais Airbus Safran Launchers est constamment à la recherche de nouvelles technologies, permettant de gagner en flexibilité et en coût, tout en limitant l'impact sur l'environnement.

La compréhension et la maîtrise des paramètres clés des sources lasers employées ainsi que l'impact sur les matériaux sont donc essentielles pour accompagner la transition vers de nouveaux procédés de préparation de surface. Cette préparation est en effet primordiale pour garantir la qualité du collage et de la protection anticorrosion.

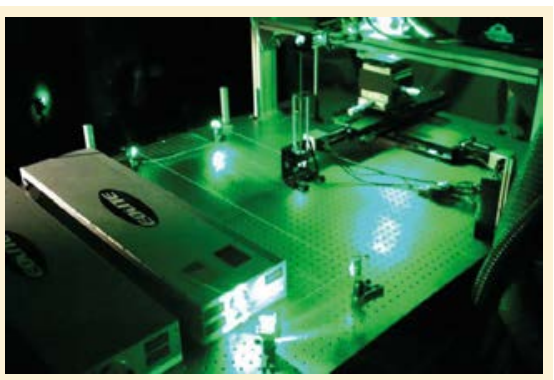

Figure 1. Banc d'essai avec source laser à $515 \mathrm{~nm}$. Cette longueur d'onde est en effet adaptée à 2 alliages étudiés au laboratoire (un alliage d'aluminium et un alliage de titane). (Crédit: Airbus Safran Launchers SAS / 2015 B. Rameau)

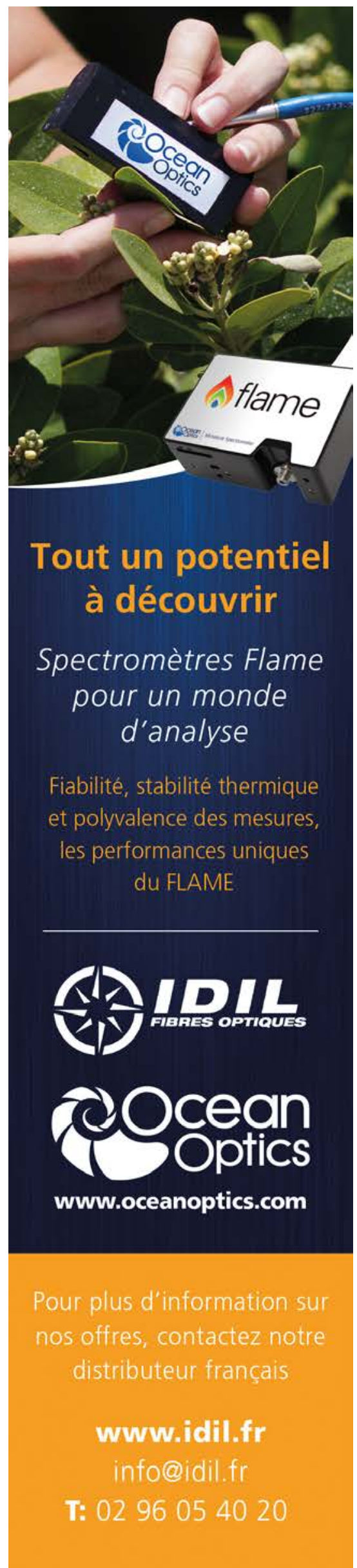

www.photoniques.com I Photoniques 84 


\section{Plan d'expérience}

ALPHANOV a étudié le potentiel du laser pour la préparation de surface avant collage ou avant protection à la corrosion. Le choix des sources s'est opéré en prenant en compte l'interaction onde-matière propice à une absorption idéale de l'onde laser (figure 1). Cette préparation par laser s'apparente à de la texturation, et les résultats sont exprimés par les caractéristiques physico-chimiques de surface suivantes: - profondeur de gravure, rugosité en fond de gravure, évolution de la mouillabilité (caractère hydrophile ou hydrophobe) des surfaces gravées au cours du temps, détection à la brûlure et caractérisation de la zone affectée thermiquement (ZAT) par analyse métallographique.

Les principaux paramètres laser étudiés (exprimés par l'énergie totale déposée en joules) sont la densité de puissance, la densité d'énergie, le nombre de passes ainsi que la distance focale.

La figure 2 illustre un exemple des aspects obtenus, dont une condition opératoire encadrée en rouge $(150 \mathrm{KHz}$ et énergie totale déposée égale à $600 \mathrm{~J}$ ) parmiles multiples testées. Pour cette condition, une rugosité arithmétique de 0,96 $\mu \mathrm{m}$ a été mesurée en fond de gravure.

\section{Pincincipaux résultats \\ Évolution de la mouillabilité}

Cette méthode consiste à mesurer l'angle de la tangente du profil d'une goutte d'eau déposée sur le substrat, avec la surface du substrat. Cet angle est appelé angle de contact et rend

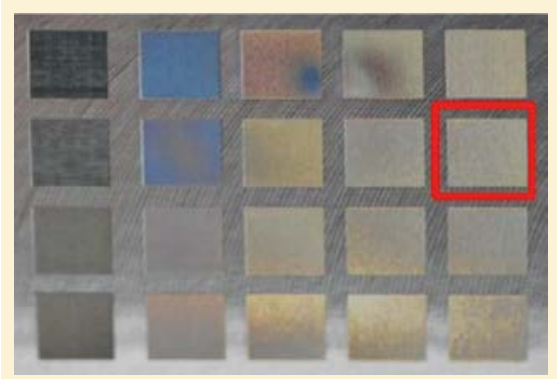

Figure 2. Zones décapées sur alliage de titane à des conditions opératoires variables. (Crédit: Airbus Safran Launchers SAS / 2015 B. Rameau)

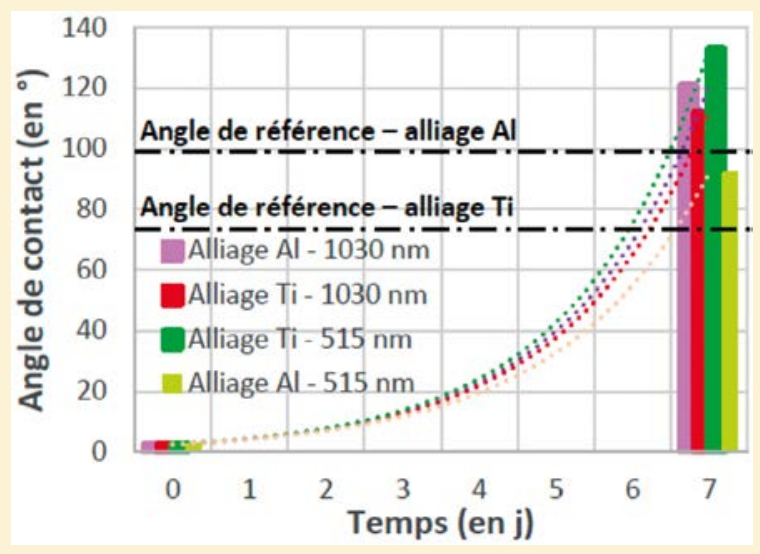

Figure 3. Évolution de l'angle de contact en fonction du temps pour les deux alliages et longueurs d'ondes, pour une énergie totale déposée égale à $600 \mathrm{~J}$.

compte du caractère hydrophile ou hydrophobe d'une surface. Pour un angle de contact inférieur à $90^{\circ}$, la surface a un caractère dit hydrophile. Pour un angle compris entre 90 et $150^{\circ}$, il est dit hydrophobe. La croissance du caractère hydrophobe d'une surface après traitement au cours du temps, est expliquée par l'oxydation de cette surface par l'atmosphère ambiante [1].

La figure 3 illustre le comportement pour deux alliages (à base titane et aluminium) et deux longueurs d'ondes laser, et pour une énergie totale déposée égale à $600 \mathrm{~J}$. Cet angle en surface du métal de base puis en surface traitée a été caractérisé à $t_{0}$ (soit immédiatement après avoir traité la surface de l'échantillon par laser) et $t_{0}+7$ jours. L'angle de contact mesuré (angle de référence) pour la surface de base est de $98^{\circ}$ pour l'alliage d'aluminium (surface à caractère hydrophobe) et de $76^{\circ}$ pour l'alliage de titane (surface à caractère hydrophile). Sur la figure 3 nous représentons ces deux angles de référence par une ligne noire pointillée.

En comparaison avec la valeur de ces angles, nous pouvons tirer les deux conclusions suivantes: (1) immédiatement après traitement par laser $\left(\right.$ à $\left.t_{0}\right)$, pour les deux alliages et deux longueurs d'ondes étudiés, nous observons un caractère hydrophile des surfaces traitées (angle de contact moyen inférieur à $\left.5^{\circ}\right) ;(2)$ il apparaît un caractère hydrophobe (plus ou moins prononcé) de ces mêmes surfaces après 7 jours. Par ailleurs, notons que la rugosité initiale en fond de gravures ne semble avoir aucune influence sur l'évolution de l'angle de contact.

\section{Analyse métallographique}

Nous avons également observé au microscope optique la structure du métal traité et nous avons mesuré la zone affectée thermiquement. Pour les deux alliages, nous avons observé une dépendance de la hauteur de cette zone avec l'énergie laser déposée. Toutefois, cette hauteur reste inférieure à $10 \mu \mathrm{m}$ par rapport à la surface pour une énergie totale déposée de 600 joules, ce qui occasionne un faible impact sur les caractéristiques mécaniques statiques des pièces (Rm et $\mathrm{A} \%$ ). Ce point est en cours d'investigation concernant les caractéristiques mécaniques en dynamique (fatigue).

\section{Conclusion}

Les conclusions de l'étude ont confirmé l'intérêt de cette technique, qui apporte une qualité de préparation de surface à la hauteur des attentes, tout en laissant entrevoir des procédés plus efficients et plus respectueux de l'environnement. Airbus Safran Launchers va donc poursuivre les études et a notamment décidé de mettre ce procédé de préparation de surface par laser en phase de qualification pour le futur lanceur Ariane 6, notamment pour des applications avant collage. Son potentiel d'application va tendre à augmenter dans les prochaines années.

\section{RÉFÉRENCE}

[1] Multifunctional surfaces produced by femtosecond Laser pulses. A.Y. Vorobyev, Chunlei Guo. The institute of Optics, University of Rochester, Rochester, New York 14627, USA, 20/01/2015. 